Interactive Effects? American Journal of Public Health 1997; 87: 1003-7.

19. Senarath U, Siriwardena I, Godakandage SSP, Jayawickrama H, Fernando DN, Dibley MJ. Determinants of breastfeeding practices: An analysis of the Sri Lanka Demographic and Health Survey 2006-2007. Maternal and Child Nutrition 2011; 8: 315-29. doi: 10.1111/j.740-8709.2011.00321.x.
20. Shekar M, Somanathan A, Du L. Malnutrition in Sri Lanka: Scale Scope, Causes and Potential Response. Washington, DC: World Bank, 2007.

21. Hui LL, Schooling CM, Cowling BJ, Leung SSL, Lam TH, Leung GM. Are universal standards for optimal infant growth appropriate? Evidence from a Hong Kong Chinese birth cohort. Archives of Disease in Childhood 2007; 93: 561-5.

\title{
Obstetric performance of women who have previously delivered a baby of low birthweight
}

\author{
H Senanayake, S Bujawansa, V Kariyawasam, H Ariyaratne \\ (Index words: previous term low birth weight, obstetric risk factors, pregnancy outcomes)
}

\begin{abstract}
Objectives To investigate pregnancy outcomes in women who have previously given birth to a baby weighing less than 2500 grams in a population in which the low birthweight rate is high.

Methods We compared the obstetric performance of 100 women who had delivered a low birthweight baby $(<2500 \mathrm{~g})$ at term in a previous pregnancy (cases) with those of 100 women who were matched for age, height, body mass index at booking, parity and medical disorders, who had previously delivered a baby above that threshold (controls).
\end{abstract}

Results We found the following significant differences between cases and controls ( $p<0.001$ for all). Gestation at delivery (mean $\pm \mathrm{SD}=38.0 \pm 1.9$ vs. $39.1 \pm 1.2$ weeks); risk of delivery before completion of 37 weeks (19.0\% vs. $3.0 \%$ ); birthweight (mean $\pm \mathrm{SD}=2.553 \pm 0.444$ vs. $3.015 \pm 0.413 \mathrm{Kg}$ ); risk of delivery of a low birthweight (LBW) baby in the current pregnancy (27.0\% vs. $4.0 \%)$. The stillbirth rate was also significantly higher among cases (4 vs. $0 ; p<0.05$ ).

Conclusions Birth of a LBW baby points to adverse events in future pregnancies even where its prevalence is high. It is a useful criterion for assessment of risk in antenatal care and for directed interventions.

Ceylon Medical Journal 2013; 58: 18-21

\section{Introduction}

Low birth weight (LBW) babies (birth weight $<2.5 \mathrm{~kg}$ ) are known to have a poor perinatal outcome and to make a disproportionate contribution to perinatal deaths $[1,2]$. In addition, they are prone to important long term complications [3].

LBW babies born at term could be considered as being small for gestational age (SGA) irrespective of ethnicity and maternal body mass index (BMI). There are a multitude of immediate and inter-generational risk factors that underlie the birth of a baby who weighs less than 2.5 $\mathrm{kg}$ at term. These include short maternal height, low maternal BMI, poor weight gain during pregnancy, maternal race, medical disorders complicating pregnancy (e.g. hypertension), substance abuse and a previous low birthweight baby. Many studies have addressed these factors, but there is little quality evidence on the future reproductive performance of women who have previously delivered LBW babies.

The majority of risk factors for LBW are likely to persist into future pregnancies and these women could potentially be at higher risk of a repeat birth of a LBW baby. Risk factors for LBW are also common to other adverse pregnancy outcomes and it is possible that the birth of a LBW baby may point to other adverse outcomes in future pregnancies. It could therefore serve as a risk factor to screen women during pregnancy.

Two previous studies have shown that women who delivered LBW babies have a greater tendency to repeat a LBW birth. However, both these studies were not restricted

Department of Obstetrics and Gynaecology, Faculty of Medicine, University of Colombo, Sri Lanka.

Correspondence: HS, e-mail: <senanayakeh@gmail.com>. Received 4 May and revised version accepted 13 October 2012. Competing interests: none declared. 
to LBW births at term and included preterm babies. Also, these studies have been done on populations that have a low background prevalence of LBW. It is known that the incidence of LBW in developing countries is more than double that of the developed countries and that the aetiology of LBW is different between developing and developed countries $[4,5]$. The main contributor in the former is fetal growth restriction, whereas in the developed countries it is prematurity [6]. Therefore findings of studies conducted on LBW in different socioeconomic settings are likely to be inapplicable across these settings.

There is no data in the literature regarding pregnancy outcomes of women who have previously delivered a LBW baby in populations that have a high LBW rates. The tendency to repeat a LBW birth may in fact be even more pronounced due to persistence of risk factors. On the contrary, it is known that birth weight increases with birth order [7]. Sri Lanka with a LBW rate of $17.3 \%$ provides a suitable setting to study this problem [8].

\section{Methods}

We studied the reproductive performance of women who previously delivered a LBW baby at term using cross sectional observational study design. Women who were admitted to the University Obstetrics Unit, De Soysa Hospital for Women, for confinement were eligible for recruitment. Women with a documented previous term LBW baby $(<2.5 \mathrm{~kg}$ at a period of gestation $>37$ completed weeks) were categorised as cases $(n=100)$. Women with no such history, matched for age (+/- 5 years), height $(+/-$ $5 \mathrm{~cm})$, body mass index at booking (BMI) $\left(+/-2.5 \mathrm{~kg} / \mathrm{m}^{2}\right)$, parity and medical disorders were selected as controls $(n=100)$. Written informed consent was obtained from the participants. Approval for this study was obtained from the Ethics Review Committee of the Faculty of Medicine, University of Colombo, Sri Lanka.

The women were recruited after the confinement of the current pregnancy. A pre-tested, structured, interviewer-administered questionnaire was used to collect data. It included sections on demographic information, known risk factors, past obstetric history and current pregnancy. To confirm gestational age, we used the results of ultrasonography performed in the second trimester. After delivery of the baby, birth weight was measured to the nearest gram within one hour of birth on an electronic measuring scale.

The Students $t$ test (2-tailed) and $\chi^{2}$ test were used to compare continuous and categorical variables respectively. Appropriate non-parametric tests were used to analyse variables that did not follow a normal distribution.

\section{Results}

The two groups were equally matched for age, height, body mass index (BMI) at booking, parity, maternal medical disorders and family income (Table 1).
Table 1. Characteristics of the study population

\begin{tabular}{lccc}
\hline Characteristic & $\begin{array}{c}\text { Previous term } \\
\text { LBW }(n=100)\end{array}$ & $\begin{array}{c}\text { Controls } \\
(n=100)\end{array}$ & $p$ \\
\hline Age (years) & $29.9 \pm 5.3$ & $30.5 \pm 4.7$ & NS \\
Height (cm) & $149.8 \pm 6.0$ & $151.0 \pm 5.7$ & NS \\
$\begin{array}{l}\text { BMI at booking } \\
\text { visit }\left(\mathrm{kg} / \mathrm{m}^{2}\right)\end{array}$ & $22.4 \pm 4.3$ & $23.3 \pm 3.5$ & $\mathrm{NS}$ \\
$\begin{array}{l}\text { Income (Rs.) } \\
\text { Recreational drug use }\end{array}$ & $0030 \pm 4756$ & $10997 \pm 10037$ & $\mathrm{NS}$ \\
$\begin{array}{l}\text { Gap after last } \\
\text { pregnancy (years) }\end{array}$ & $4.0 \pm 2.4$ & $4.3 \pm 3.3$ & $\mathrm{NS}$ \\
POG at booking (weeks) & $17.7 \pm 6.4$ & $18.1 \pm 5.7$ & $\mathrm{NS}$ \\
No of clinic visits & $6 \pm 2$ & $7 \pm 2$ & $\mathrm{NS}$ \\
\hline
\end{tabular}

$\mathrm{LBW}=$ Low birth weight, $\mathrm{POG}=$ Period of gestation, $\mathrm{NS}=$ Not significant Data shown as mean $\pm 1 \mathrm{SD}$

There were no significant differences in the period of gestation at referral, number of clinic visits or mode of delivery.

A comparison of the reproductive performance of the two groups is shown in Table 2.

Table 2. Pregnancy outcome of the two groups

\begin{tabular}{lccc}
\hline Outcome & $\begin{array}{c}\text { Previous term } \\
\text { LBW }(n=100)\end{array}$ & $\begin{array}{c}\text { Controls } \\
(n=100)\end{array}$ & $p$ \\
\hline $\begin{array}{l}\text { Gestation at delivery } \\
\text { (weeks) }\end{array}$ & $38.0 \pm 1.9$ & $39.1 \pm 1.2$ & $<0.001$ \\
Delivery $<37$ weeks & $19.0 \%$ & $3.0 \%$ & $<0.001$ \\
Birth weight $(\mathrm{kg})$ & $2.553 \pm 0.444$ & $3.015 \pm 0.413$ & $<0.001$ \\
Birth weight $<2.5 \mathrm{~kg}$ & $27.0 \%$ & $4.0 \%$ & $<0.001$ \\
at term & $4.0 \%$ & $0.0 \%$ & $<0.05$ \\
Stillbirths & $1.0 \%$ & $1.0 \%$ & $\mathrm{NS}$ \\
Neonatal deaths & $8.0 \%$ & $4.0 \%$ & $\mathrm{NS}$ \\
SCBU admission & & & \\
\hline
\end{tabular}

SCBU $=$ Special Care Baby Unit

We found significant differences in the gestation at delivery, risk of delivery before completion of 37 weeks, birthweight and risk of delivery of a low birthweight baby in the current pregnancy ( $p<0.001$ for each). The stillbirth rate was also significantly higher among cases $(p<0.05)$.

\section{Discussion}

In populations that have a high prevalence of $\mathrm{LBW}$, the birth of such a baby is often taken for granted as an insignificant event. However, this study shows that it is an important pointer to adverse outcomes in future pregnancies. 
To our knowledge, this is the first study on this subject that was conducted in a population with a high incidence of LBW. In addition, we addressed weaknesses in other studies by studying women who delivered LBW babies at term rather than preterm and by controlling the comparison group for age, parity, height and medical disorders and by controlling the two populations for multiple factors. Other investigators had not adjusted for other known risk factors $[9,10,11]$. One study included mothers with previous preterm deliveries and merely demonstrated a statistically significant reduction in birth weight that was nevertheless within the normal range [11].

This study demonstrates clinically relevant differences in late pregnancy outcomes of women who have had a previous LBW birth, highlighting its importance as a risk factor even in the presence of a high background prevalence. There was a significant risk of delivering another low birth-weight baby in a future pregnancy $(27 \%$ vs. $4 \% ; p<0.001)$. The risk of other adverse outcomes such as preterm labour in subsequent pregnancies was also increased. There was also a clear trend towards other perinatal complications. Both stillbirths and Special care baby unit admissions were increased. Among the controls in the study there were no stillbirths compared to a rate of $4 \%$ among the cases $(p<0.05)$. These results have important implications in risk assessment.

The association between the birth of a previous LBW baby at term and the subsequent risk of adverse pregnancy outcomes may be explained by the fact that common factors underlie these events. Inter-generational research suggests that there may be genetic determinants of reproductive outcomes [13]. Genetic and environmental factors that contribute to improper development of the placenta and thereby to fetal growth restriction and placental insufficiency could continue their influence from one generation to another leading to adverse perinatal events. The relationship between the birthweights of the mother and her offspring is well recognised $[14,15]$. There is also data that links a mother who was born with a low birth weight with development of pre-eclampsia and gestational diabetes in adulthood $[16,17]$.

Conversely, the birth of a baby of normal weight could be considered a surrogate marker of the absence or the ability of the maternal physiology to override them. Among the controls, who had previously delivered a baby of normal birthweight, the incidence of LBW was $4 \%$.

The strong effect of maternal height and BMI at booking, on birth weight was realised during the course of the study, as matching controls for these factors proved difficult. This is again, a reflection of the long-term factors that affect LBW. Unmatched grouping was used in a previous study done in the US [10]. Interestingly, there were no significant differences in maternal height or weight between the two groups in the US study. However, factors such as tobacco and alcohol use were significantly commoner in American mothers with a LBW baby. In our study population, substance abuse among pregnant women was nonexistent, highlighting differences in factors that influence the risk for LBW in different settings.

This study illustrates the importance of a previous birth of a LBW baby as a risk factor that must be looked for in the routine antenatal history and risk assessment, even in populations with a high prevalence of the condition. This will help recognize a subset of mothers who are at risk of adverse pregnancy outcomes.

There is a popular belief that there are no interventions that could improve birthweight, other than advising against smoking and substance abuse. However, in populations with poor nutrition there may be more that could be done. Poor weight gain during pregnancy and pre-pregnancy weight have been recognized as factor having a strong influence on LBW [18]. Thus, it may be possible to intervene in women who deliver LBW babies before their next pregnancy, such as by improving prepregnancy weight. Also, they could be targeted for nutritional advice aimed at improving the weight gain during pregnancy. Such interventions could potentially help reduce LBW rates. In Gambia, an improvement in birthweight with nutritional supplementation was demonstrated, with the effect being most pronounced in the severely undernourished (19). However, there is no evidence at present to suggest that nutritional interventions could override the influence of long-term risk factors for LBW. This may be worthy of further research.

In the modern context, the use of a previous birth of a LBW baby as a screening measure could enable such women to be selected for screening by methods such as uterine artery doppler assessment in mid pregnancy to recognise the risk of placental insufficiency.

Our findings are contrary to the widely held belief that pregnancy performance improves with birth order, underscoring the influence and constancy of risk factors for LBW and their association with other adverse pregnancy outcomes. A history of birth of a LBW baby in the past is a risk factor that shows that the mother requires nutritional advice and targeted monitoring in subsequent pregnancies for serious adverse events.

\section{References}

1. Lucas GN, Ediriweera RC. Perinatal deaths at the Castle Street Hospital for Women in 1993. Ceylon Medical Journal 1996; 41:10-2.

2. Fonseka P, Wijewardene K, de Silva HDG, Goonaratna C, Wijeyasiri WA. Neonatal and post-neonatal mortality in the Galle district. Ceylon Medical Journal 1994; 39: 82-5.

3. Barker DJ. The origins of the developmental origins theory. Journal of Intern Medicine 2007; 261: 412-7.

4. Ohlsson A, Shah P. Determinants and Prevention of Low Birth Weight: A Synopsis of the Evidence. Alberta, Canada: Institute of Health Economics. 2008.

5. Ramakrishnan U. Nutrition and low birth weight: from 
research to practice American. Journal of Clinical Nutrition 2004; 79: 17-21.

6. Kramer MS. Determinants of low birth weight: methodological assessment and meta-analysis. Bulletin of the World Health Organization 1987; 65: 663-737.

7. Nanayakkara KK, Samarakoon AB, Perera BH, et al. Size of newborns at birth. Journal of Obstetrics and Gynaecology Research 2011; 31: 311-4.

8. Annual Health Statistics Sri Lanka 2007. Medical Statistics Unit, Department of Health, Ministry of Health and Nutrition of Sri Lanka. 2008. P54.

9. Freeman MG, Graves WL. Risk of premature delivery among indigent Negro women based on past reproductive performance. Obstetrics and Gynaecology 1969; 34: 648-54.

10. Kaltreider DF, Johnson JWC. Patients at high risk for lowbirth-weight delivery. American Journal of Obstetrics and Gynecology 1976; 124: 251-6.

11. Tejani NA. Recurrence of intrauterine growth retardation. Obstetrics and Gynecology 1982; 59: 329-31.

12. Goldenberg RL, Hoffman HJ, Cliver SP, Cutter GR, Nelson KG, Copper RL. The Influence of Previous Low Birth Weight on Birth Weight, Gestational Age, and Anthropometric Measurements in the Current Pregnancy. Obstetrics and Gynecology 1992; 79: 276-9.

13. Emanuel I. Invited commentary: an assessment of maternal intergenerational factors in pregnancy outcome. American Journal of Epidemiology 1997; 146: 820-5.

14. Emanuel I, Leisenring W, Williams MA, et al. The Washington State Intergenerational Study of Birth Outcomes: methodology and some comparisons of maternal birthweight and infant birthweight and gestation in four ethnic groups. Paediatric and Perinatal Epidemiology 1999; 13: 352-69.

15. Farina A, Dini B, Mattioli M, Rosa S, Rizzo N. Offspring birth weight in second-generation 'small for gestational age' infants. Prenatal Diagnosis 2010; 30: 551-4.

16. Dempsey JC, Williams MA, Luthy DA, Emanuel I, Shy K. Weight at birth and subsequent risk of preeclampsia as an adult. American Journal of Obstetrics and Gynecology 2003; 189: 494-500.

17. Rogvi R á, Forman JL, Damm P, Greisen G. Women Born Preterm or with Inappropriate Weight for Gestational Age Are at Risk of Subsequent Gestational Diabetes and PreEclampsia. PLoS One 2012; 7: e34001.

18. World Health Organisation. Maternal anthropometry and pregnancy outcomes: a WHO collaborative study. Bulletin of the World Health Organization 1995; 73 (Supp): 1-98.

19. Ceesay SM, Prentice AM, Cole TJ, et al. Effect on birth weight and perinatal mortality of dietary supplements in rural Gambia: 5 year randomized controlled trial. British Medical Journal 1997; 315: 786-90.

\section{Tuberculous meningitis in adults: a prospective study at a tertiary referral centre in Sri Lanka}

\section{S A C U Gunawardhana, S C Somaratne, M A H Fernando, P S Gunaratne}

(Index words: tuberculous meningitis, clinical presentation, magnetic resonance imaging, Sri Lanka)

\begin{abstract}
Introduction Central nervous system tuberculosis is the most severe form of extrapulmonary TB and it is associated with a substantial morbidity and mortality.

Objectives To describe the demographic profile, clinical features, laboratory and imaging results of a cohort of adult patients with TBM (Tuberculous meningitis).

Methods This study encompasses a prospective analysis of all adult cases of TBM diagnosed from 1st January 2010 to 31st December 2011 in the Neurology unit 2, National Hospital of Sri Lanka. Consensus case defini-
\end{abstract}

tions for TBM were used for clinical case classification and patients were given a definite, probable, or possible tuberculous meningitis status accordingly.

Results A total of 89 patients fulfilled the established diagnostic criteria for TBM and there were 22 definitive cases, 46 probable cases and 21 possible cases. The mean age of the series was 44 years and $56(63 \%)$ were males. TBM presented with fever in $64(71 \%)$, general constitutional symptoms in $61(68 \%)$, headache in 53 (59\%), and diminished level of consciousness in 36 (40\%) patients. CSF biochemistry revealed elevated protein in all patients. MRI brain showed meningeal

Unit 2, Institute of Neurology, National Hospital of Sri Lanka.

Correspondence: SACUG, e-mail: <champikagu@yahoo.com>. Received 10 July and revised version accepted 11 October 2012. Competing interests: none declared. 